\title{
Inflow Cannula Positioning of the HeartWare Device is Not Associated with Thomboembolic Events
}

\author{
Nicholas Hess ${ }^{1}$, Edgar Aranda-Michel ${ }^{2}$, Gavin Hickey ${ }^{2}$, Yisi Wang ${ }^{2}$, Floyd Thoma ${ }^{2}$, and \\ Arman Kilic ${ }^{2}$ \\ ${ }^{1}$ University of Pittsburgh Medical Center \\ ${ }^{2}$ University of Pittsburgh Medical Center Health System
}

July 11, 2020

\begin{abstract}
BACKGROUND Prior studies have suggested that LVAD implantation technique and positioning may be associated with subsequent thromboembolic complications. This study evaluated the impact of inflow cannula positioning of the HeartWare LVAD (HVAD) on clinical outcomes. METHODS Adults undergoing primary HVAD implantation between 2009-2018 at a single center were reviewed. HVAD position parameters were calculated from plain chest radiographs and included cannula angle, pump depth, and pump area. These were measured at the day of HVAD implant, at 1 month post-HVAD, and at 6 months postHVAD. Changes in these parameters over time were measured. Multivariable analysis was also conducted to evaluate associations between pump position parameters on the day of implant and one-year composite outcome of thromboembolic complication (embolic stroke or pump thrombosis) and death. RESULTS 159 HVAD recipients were included with a median follow up of 2.6 years (IQR 1.3 - 4.8 years). Pump area decreased from immediate post-HVAD implantation to one-month follow up (-0.7 $\mathrm{cm} 2 ; \mathrm{p}=0.002)$, but no significant changes were noted with other position parameters. Increasing preoperative serum creatinine level (HR 1.46, 95\% CI $1.03-2.08, \mathrm{p}=0.034$ ) was associated with increased hazards for the composite outcome in multivariable analysis. Cannula angle, pump depth, nor pump area were associated with the one-year composite outcome, readmission, or other one-year complications including thromboembolic events or mortality when separately modeled. CONCLUSION This review of HVAD recipients demonstrates device position as assessed by chest X-ray does not have significant predictive associations with one-year outcomes.
\end{abstract}

\section{Hosted file}

HVAD_Position_JCardSurg_7_10_flat.doc available at https://authorea.com/users/331307/ articles/468772-inflow-cannula-positioning-of-the-heartware-device-is-not-associatedwith-thomboembolic-events 


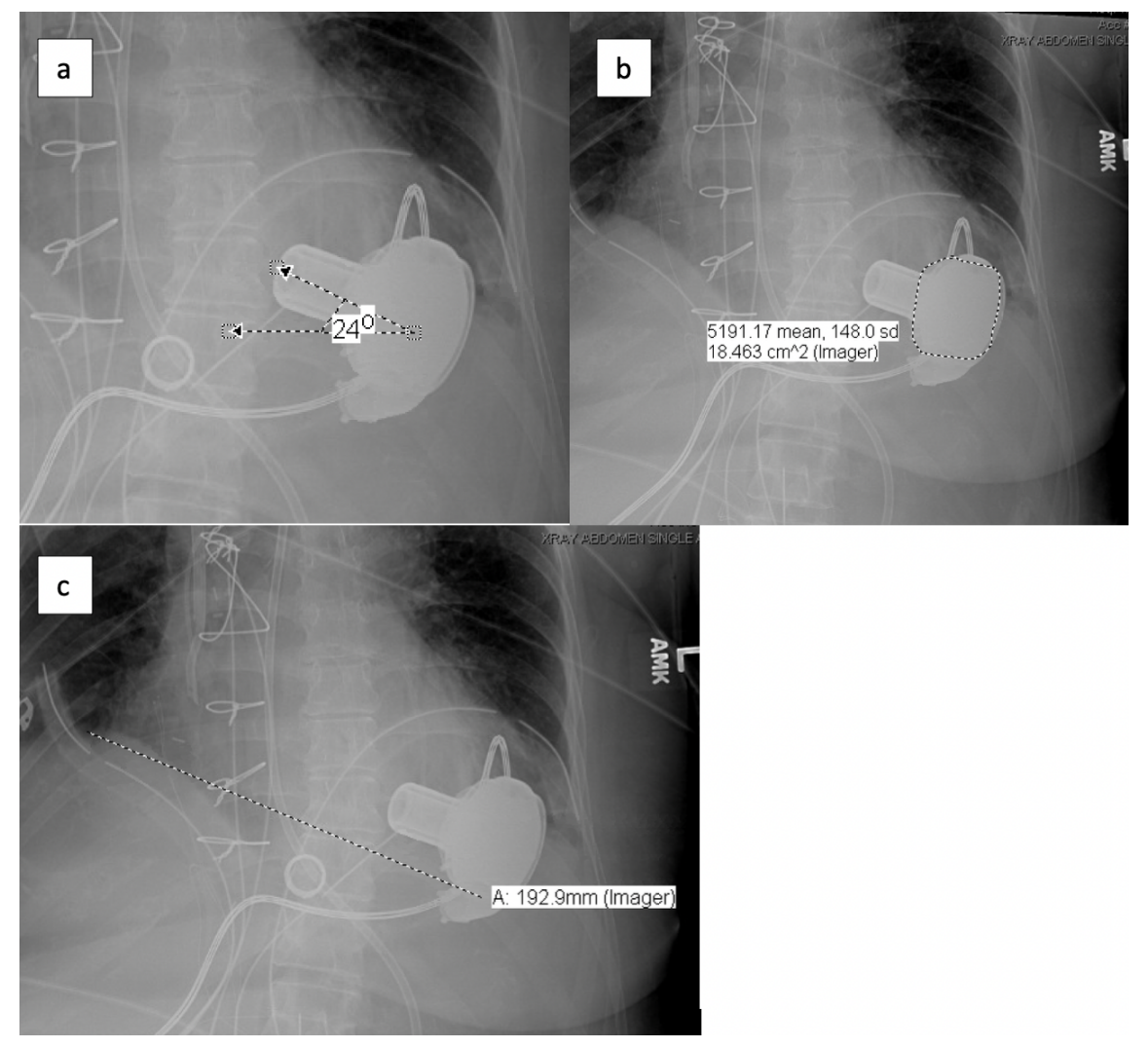

